\title{
Now You See It, Now You Don't: Testing Environments Modulate the Association Between Hippocampal Volume and Cortisol Levels in Young and Older Adults
}

\author{
Shireen Sindi, ${ }^{1,2}$ Alexandra J. Fiocco, ${ }^{3}$ Robert-Paul Juster, ${ }^{1,2}$ Catherine Lord, ${ }^{1}$ \\ Jens Pruessner, ${ }^{4,5}$ and Sonia J. Lupien ${ }^{1,6 *}$
}

\begin{abstract}
The hypothalamic pituitary adrenal axis production of the stress hormone cortisol interacts with the hippocampal formation and impacts memory function. A growing interest is to determine whether hippocampal volume (HV) predicts basal and/or reactive cortisol levels in young and older adults. Recent evidence shows that contextual features in testing environments might be stressful and inadvertently induce a stress response in young and/or older populations. This latter result suggests that variations in testing environments might influence associations between HV and cortisol levels in young and older adults. To this end, we investigated 28 healthy young adults (ages 18-35) and 32 healthy older adults (ages 60-75) in two different environments constructed to be more or less stressful for each age group (Favoring-Young versus Favoring-Old conditions). Cortisol levels were repeatedly assessed in each environment, and young and older participants underwent an anatomical magnetic resonance imaging scan for subsequent assessment of HV. Results in both age groups showed that HV was significantly associated with cortisol levels only in the unfavorable stressful testing conditions specific for each age group. This association was absent when testing environments were designed to decrease stress for each age group. These findings are fundamental in showing that unless the nature of the testing environment is taken into consideration, detected associations between HV and cortisol levels in both young and older populations might be confounded by environmental stress. $\odot 2014$ Wiley Periodicals, Inc.
\end{abstract}

KEY WORDS: hippocampal volume; cortisol; testing environment; aging; stress reactivity; basal cortisol

\footnotetext{
${ }^{1}$ Centre for Studies on Human Stress, Mental Health Research Centre Fernand Seguin, Hospital Louis H Lafontaine, Montreal, Quebec, Canada; ${ }^{2}$ Department of Neurology and Neurosurgery, McGill University, Montreal, Quebec, Canada; ${ }^{3}$ Department of Psychology, Ryerson University, Toronto, Ontario, Canada; ${ }^{4}$ Douglas Mental Health University Institute, McGill University, Montreal, Quebec, Canada; ${ }^{5}$ McGill Centre for Studies in Aging, Faculty of Medicine, McGill University, Montreal, Quebec, Canada; ${ }^{6}$ Department of Psychiatry, Faculty of Medicine, Université de Montréal, Montreal, Quebec, Canada.

Grant sponsor: Canadian Institutes of Health Research; Grant number: 77788; Grant sponsor: Frederick Banting and Charles Best Canada Graduate Doctoral Scholarship (awarded by the Canadian Institutes of Health Research).

*Correspondence to: Sonia Lupien, Centre for Studies on Human Stress (www.humanstress.ca), 7331 Hochelaga, Montreal, Quebec, H1N 3V2Canada. E-mail: sonia.lupien@umontreal.ca
}

\section{INTRODUCTION}

The hypothalamic-pituitary-adrenal (HPA) axis and its end-products [glucocorticoids (GCs); cortisol in humans] play a major role in stress responsivity. GCs are liposoluble and cross the blood-brain barrier to modulate receptive brain regions, including the hippocampus (Lupien and McEwen, 1997; de Kloet et al., 2005; McEwen, 2007). Whereas cortisol levels are associated with hippocampal volume (HV) among various populations (reviewed by Frodl and O'Keane, 2013), it is important to distinguish basal from reactive levels. Basal cortisol levels represent daily secretion, which follows a circadian rhythm. Upon awakening, the cortisol awakening response (CAR) occurs, and levels steadily decline throughout the day (Fries et al., 2009). In contrast, reactive cortisol levels are induced by acute stressors (Kirschbaum et al., 1993; Dickerson and Kemeny, 2004).

Studies in psychiatric patients report associations between HPA axis dysregulation and reduced HV (O'Brien et al., 2004; Swaab et al., 2005; Bremner, 2006; Karl et al., 2006). Among healthy populations, studies have revealed that smaller HV is associated with higher basal cortisol levels in young (Wolf et al., 2002; Vythilingam et al., 2004) and older adults (Lupien et al., 1998; Wolf et al., 2002; O'Hara et al., 2007; Bruehl et al., 2009; Knoops et al., 2010). However, results elsewhere show no such association in various age groups (age range: 20-70 years) (MacLullich et al., 2005; Gunduz-Bruce et al., 2007; Gold et al., 2010; Kaymak et al., 2010; Kremen et al., 2010; Mondelli et al., 2010; Collip et al., 2013). Caution must therefore be taken when drawing conclusions based on the current literature. A recent review by Frodl and O'Keane (2013) concluded there are no evident methodological differences between studies, so discrepancies might be due to chance. For these authors, the mixed findings may be explained by the number of basal cortisol samples measured; studies using more samples are more likely to report significant associations between $\mathrm{HV}$ and basal cortisol levels (Frodl and O'Keane, 2013).

Despite Frodl and O'Keane's point of view, another possibility that might explain these discrepancies is 
participants' stress reactivity during basal measurements. Here, contextual features within testing environments may induce a spurious reactive cortisol response. This implies that "basal cortisol levels" could in fact represent a "reactive cortisol response" (Sindi et al., 2013).

Testing environments may be distressing when they inadvertently include psychological factors known to elicit stress responsivity, to which older adults are sensitive (novelty, unpredictability, threat to the ego, and a diminished sense of control) (Mason, 1968; Nicolson et al., 1997; Dickerson and Kemeny, 2004; Lupien et al., 2007a). Our team recently investigated whether manipulating stressful factors within testing environments (e.g., non-optimal testing time, unfamiliarity with testing location, young age of research assistant and type of unfamiliar cognitive task; see Lupien et al., 1997, 2007b) might modulate cortisol levels and memory performance. To do so, young and older participants were tested in two environments modified to be more or less stressful for each age group: "Favoring-Young condition" and "Favoring-Old condition." Results showed that only when older adults were tested in the Favoring-Young condition, they secreted high cortisol levels and showed impaired memory performance. This suggests that older adults' sensitivity to their surroundings artificially increases cortisol levels and impairs cognition (Sindi et al., 2013).

Consistently, whether a significant association between HV and basal cortisol levels presents in any given study may depend on participants' reactivity to the testing environment. A testing environment that is inherently stressful could increase "basal" cortisol levels and induce a significant correlation with HV. Since as an age group, older adults have smaller HV when compared to young adults, it is unclear whether this renders them more sensitive to testing environments (Van Petten, 2004). It is therefore important to assess associations between $\mathrm{HV}$ and cortisol levels in both young and older adults in favorable and unfavorable environments. The goal of this study was to determine whether within the same age group, associations between $\mathrm{HV}$ and cortisol levels differ as a function of reactivity to the testing environment.

\section{METHODS}

\section{Participants}

The sample consisted of 28 young adults (nine men, 19 women) between the ages of 18 and $35(\mathrm{M}=24$, S.E.M $=$ 0.87 ) and 32 healthy older adults (14 men, 18 women) between the ages of 60 and $75(\mathrm{M}=67$, S.E.M $=0.82)$. Young adults were recruited from the campus of McGill University in Montreal, Canada, while older adults were recruited from advertisements in various newspapers published in Montreal, Canada. Young adults were university students, with a mean of 15 years of education (S.E.M = 0.27). Likewise, older adults had a mean of 14 years education (S.E.M $=0.56$ ). All
A

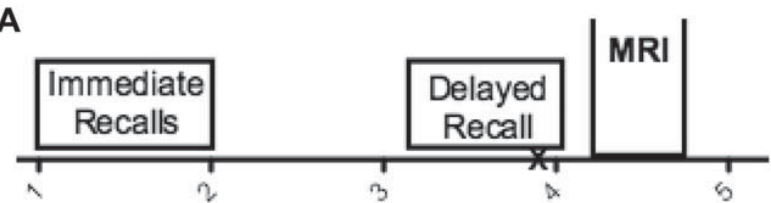

B

Cortisol Sample

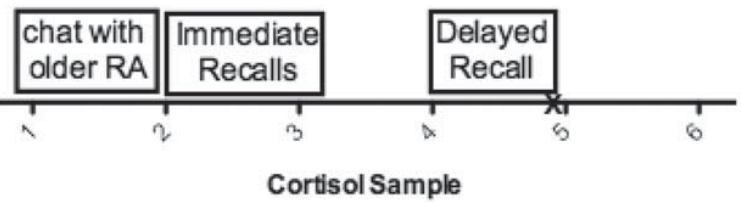

FIGURE 1. A: Graphic representation of the saliva sampling time points in the Favoring-Young condition. B: Graphic representation of the saliva sampling time points in the Favoring-Old condition.

participants of this study were part of a larger study and we have previously published findings on cortisol reactivity to variations in testing environments using the same sample of participants (Sindi et al., 2013).

Prior to recruitment, participants were called for a structured phone screening, where they were asked to self-report whether they have any medical or psychiatric conditions. Participants were asked about cardiovascular and endocrine conditions, diabetes, use of medications that impact the HPA axis, body mass index, neurological conditions, head trauma, and recent general anaesthesia. Participants were also screened for depressive symptoms and cognitive impairment. Individuals who had mentioned having experienced a major life event in the past year were not included. Only older women who were not using hormone replacement therapy were recruited for the study. Participants provided written informed consent prior to their participation in the study. The Research Ethics board of the Douglas Mental Health University Institute approved the study, which adheres to the declaration of Helsinki.

\section{MATERIAL AND METHODS}

Both older and young adults were each tested in two testing environments manipulated to be more or less stressful for their age group. The environments differed in terms of the following factors: (1) location of testing, (2) age of research assistant, (3) time of testing and (4) instructions/type of task. A detailed description of the content of the memory tasks and for the rationale of these factors as modulators of the stress response to testing environments has been recently described in detail elsewhere (Sindi et al., 2013). The first condition was a testing environment that was favorable for young individuals (thereafter called the "Favoring-Young condition"). The second condition was a testing environment that was favorable for older individuals (thereafter called the "Favoring-Old condition"). All participants (young and older adults) were exposed to both 
conditions (Favoring-Young and Favoring-Old). The conditions were counterbalanced within and across the two age groups.

\section{Favoring-Young condition}

This session took place in the afternoon, between noon and 16:00 at McGill University's Montreal Neurological Institute. While this location is well known to McGill University students, it is not very familiar to older adults. Participants were met by a young graduate student (24-years-old) who administered a wordlist recall task. The task consisted of an immediate recall phase, and a delayed recall 20 min later. Participants then underwent a magnetic resonance imaging (MRI) scan. To measure cortisol levels, saliva samples were collected at 10-min intervals throughout the session (for a graphic representation of the saliva sampling time points, see Fig. 1A). This testing environment was considered favorable for young adults (and consequently, unfavorable for older adults) because it was performed in a familiar location with a young research assistant, who administered a task that is routine to young university students (word-list recall), in the afternoon, a time of testing that has been shown to be less favorable for older adults when compared to young adults (Sindi et al., 2013).

\section{Favoring-Old condition}

This session took place in the morning, between 8:00 and 11:00 am at the Douglas Mental Health University Institute. Prior to the first testing session, older adults were invited to this location to attend a non-testing visit to acclimatise participants to the environment, have them meet the research assistants and learn about what the study entails. In contrast, young individuals were not invited to this acclimatisation session, nor were they given instructions on how to get to the Douglas Mental Health University Institute, which is located $13 \mathrm{~km}$ away from McGill University and difficult to access by public transport. Participants were met by an older research assistant (72-years-old), who chatted with them during the first 10 -min rest period. She then administered a face-association memory task. The task consisted of an immediate recall phase, and a delayed recall 20 min later. Throughout the testing session, saliva samples were collected at 10-min intervals for measures of cortisol concentration (for a graphic representation of the saliva sampling time points, see Fig. 1B). This testing environment was considered favorable for older adults (and consequently, unfavorable for young adults) because it was performed in the morning, in a location familiar to older adults but not to young adults, and was carried out by an older research assistant.

\section{Subjective ratings for preference of testing environment}

The following question was used to assess participants' subjective preferences for the Favoring-Young and the FavoringOld testing conditions: "Which environment did you feel more comfortable about visiting? [a] The Montreal Neurological Institute, or [b] The Douglas Hospital."
Note that [a] is where the Favoring-Young condition took place and $[\mathrm{b}]$ is where the Favoring-Old condition took place.

\section{Measures of cortisol levels sampled at home}

Participants were instructed to sample their saliva on two weekdays in their home or natural environment. Samples were collected at the following time points: (1) awakening, (2) awakening +30 min, (3) 14:00, (4) 16:00, and (5) before bedtime. Participants were also instructed to use logbooks and document exact sampling time. Participants stored the samples in their home freezer and brought the samples to their following laboratory visit. Upon assaying, analyses used raw cortisol values, and for each time point, the average of both sampling days was calculated.

\section{Measures of cortisol levels sampled at the testing environments}

In each testing environment, saliva samples were collected throughout the session at ten-minute intervals to measure cortisol levels in response to the testing environment. In the Favoring-Young condition, a total of five samples were collected at $10 \mathrm{~min}$ intervals, including pre- and post-scan cortisol samples to determine whether the MRI procedure induced a stress response. For the Favoring-Old condition, six samples were collected at $10 \mathrm{~min}$ intervals (For descriptive statistics of the cortisol measures see Table 1).

\section{Cortisol analyses}

All saliva samples were collected using small plastic vials (passive drool). To avoid contamination, participants were asked not brush their teeth or eat immediately before saliva collection. Samples were stored at $-20^{\circ} \mathrm{C}$ until they were assayed. Analyses were performed at the Centre for Studies on Human Stress (Montreal, Canada; www.humanstress.ca) using Enzyme Immunoassay kits from Salimetrics LLC (PA).

\section{MRI image acquisition and processing for measures of $\mathrm{HV}$}

Participants were scanned in a 1.5-T Siemens Magnetom Sonata Vision scanner at the Montreal Neurological Institute (Montreal, Canada). A standard three-dimensional gradientecho pulse sequence was used, with a field of view of $256 \mathrm{~mm}$, isotropic voxel size of $1 \mathrm{~mm}$, repetition time of $22 \mathrm{~ms}$, echo time of $9.2 \mathrm{~ms}$, and flip angle of $30^{\circ}$.

Prior to manual segmentations of $\mathrm{HV}$, anatomic images were processed for corrections of intensity non-uniformity (Sled et al., 1998). To correct for differences in head size (Collins et al., 1994), the International Consortium for Brain Mapping 152 model brain was used to register images into the Montreal Neurological Institute normalised brain template (Evans et al., 1994). Manual volume segmentations of HV were ascertained using the software DISPLAY (McConnell Brain Imaging Centre, Montreal, Canada), which allows for simultaneous viewing of the brain and the region of interest in all three orientations (Pruessner et al., 2000). The manual HV segmentation protocol and the 
anatomical boundaries of the structure have been previously described in detail (Pruessner et al., 2000). All HV segmentations were performed by an experienced rater, who was blind to participants' characteristics. The intra-rater reliability coefficient was above 0.92 . With regard to the brain imaging analyses performed, the results were automatically corrected for total brain and head volumes, as all images were registered into standard stereotaxic space prior to statistical analysis. This step automatically corrects for any inter-individual differences in head and brain size so that later comparisons are unaffected by variations in head/ brain size existing between the individuals. Bivariate correlations revealed that right and left HV were highly correlated among young $(r=0.915, P=0.0001)$ and older adults $(r=0.866, P$ $=0.0001)$. Consequently, volumes of right and left hippocampi were combined into a composite score.

\section{ANALYSES}

\section{Subjective Preference for the Different Testing Conditions}

To determine the subjective preference of young and older adults for the Favoring-Young and the Favoring-Old conditions, we performed a $\operatorname{chi}^{2}\left(\chi^{2}\right)$ test, in which columns are age groups (young and older adults) and rows were selections of testing environment preference (Favoring-Young and the Favoring-Old conditions).

\section{Cortisol Levels Sampled at Home}

Considering that previous studies have differed on whether associations between $\mathrm{HV}$ and cortisol levels were found for the CAR, PM cortisol, or total levels, analyses were performed using different measures of the circadian profile. Measures of area under the curve (AUC) are optimal for usage with data collected in controlled laboratory environments during a relatively short period (Pruessner et al., 2003), yet alternative measures are available to capture circadian variation patterns across the day, while removing the influence of factors that impact a single value. Moreover, the CAR is a response to awakening, and as suggested by Clow et al. (2010), it is therefore more appropriate to use a measure of change rather than total AUCg. We therefore used the following three scores to capture inter-individual differences in circadian variation: (1) CAR, (2) PM cortisol levels, and (3) maximum-minimum cortisol levels.

\section{CAR}

The value of the awakening cortisol levels were subtracted from the value of awakening cortisol levels $+30 \mathrm{~min}$ to provide a measure of change.

\section{PM cortisol levels}

The average of the 14:00, 16:00 and bedtime cortisol values were calculated.

\section{Maximum-minimum cortisol levels}

Considering that maximum cortisol levels are reached $30 \mathrm{~min}$ after awakening, while minimum levels are reached approximately at midnight, the bedtime cortisol value was subtracted from the awakening $+30 \mathrm{~min}$ value to reflect the cortisol amplitude.

\section{Cortisol Levels Sampled at the Testing Environments}

To assess cortisol concentration, we used the trapezoidal method to calculate AUC with respect to ground (AUCg) and AUC with respect to increase (AUCi) for the Favoring-Young condition (samples 1-6), and the Favoring-Old condition (samples 1-5), respectively (Pruessner et al., 2003). AUCg incorporates the change between samples across time and their distance from the ground (zero), which is the first baseline sampling point (Pruessner et al., 2003). The AUCi also measures change across time, but it differs in not taking into consideration the change from baseline for all measurement samples (Pruessner et al., 2003). Due to this difference, associations between single sample measurements and AUCg are stronger than those observed for AUCi (Pruessner et al., 2003).

\section{Cortisol Levels in Response to the MRI Scan}

Given that the MRI scan has been shown to induce a cortisol stress response in young participants (Tessner et al., 2006), we first determined whether the scan increased cortisol levels in young and older adults. A mixed-design ANOVA was performed with age group (young vs. older adults) as the between subjects factor and time (cortisol levels pre- and post-MRI scan) as the repeated measures factor.

\section{HV in Young and Older Adults}

$\mathrm{HV}$ and variance in $\mathrm{HV}$ were compared between young and older adults using an ANCOVA, covarying for age and education and following with a Levene's test for homogeneity of variances.

\section{Associations Between HV and Cortisol Levels Measured at Home}

To determine whether HV was associated with the different measures of basal diurnal cortisol levels (CAR, PM cortisol levels, maximum-minimum cortisol levels), for each age group, partial correlational analyses were performed between HV and each of the three diurnal measures, using age and education as covariates.

\section{Associations Between HV and Cortisol Levels Measured at the Testing Environments}

To assess whether the associations between HV and cortisol levels differ as a function of the testing environment, for each age group partial correlations were performed between $\mathrm{HV}$ and 


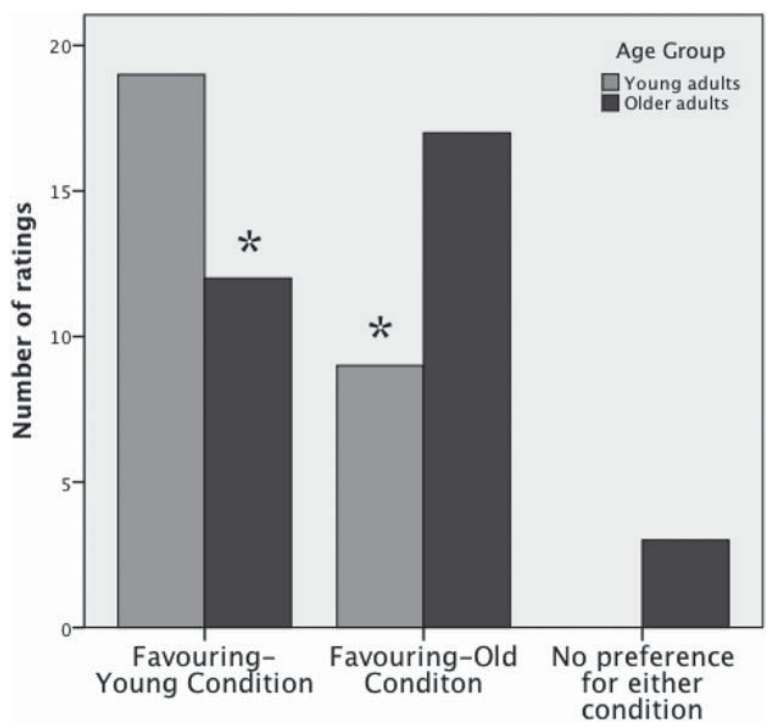

FIGURE 2. Subjective preference for the different testing conditions among young and older adults.

AUCg and AUCi cortisol levels for each testing environment, controlling for age and education.

\section{RESULTS}

\section{Cortisol Levels in the Different Testing Environments}

Among older adults, cortisol levels at the Favoring-Young conditions were significantly higher than time-matched afternoon home cortisol levels $(P<0.009)$. In contrast, in the Favoring-Young condition, results revealed that the cortisol levels secreted by young adults did not significantly differ from time-matched afternoon home cortisol levels. In the FavoringOld condition for both young and older adults, there were no significant differences between cortisol levels measured at the Favoring-Old condition compared to time-matched morning home cortisol levels (Sindi et al., 2013).

\section{Subjective Preference for the Different Testing Conditions Among Young and Older Adults}

Results of the $\chi^{2}$ test revealed there were significant differences between young and older adults' subjective preference for the testing environments that were specifically designed to be more or less favorable for each group. Young adults were more likely to prefer the Favoring-Young condition, whereas older adults were more likely to prefer the Favoring-Old condition $\left[\chi^{2}=(2\right.$, $N=60)=6.8, P=0.03]$, as can be seen in Figure 2.

\section{Cortisol Levels in Response to the MRI Scan}

Results of the mixed-design ANOVA showed that there was no effect of time $[F(1,56)=1.10, P=0.30]$, no significant effect of age group $[F(1,56)=3.13, P=0.08]$, and no interaction effect between age group and time $(P>0.33)$, indicating that the MRI scan did not induce a significant stress response in either age group. Therefore, all cortisol samples were used to calculate the AUC for cortisol secretion.

\section{HV in Young and Older Adults}

Results of the ANCOVA showed that young adults presented significantly larger $\mathrm{HV}$ compared to older adults $[F(1,53)=$ 4.59, $P=0.04]$. This difference was approximately $1 \mathrm{~cm}^{3}$, as can be seen in Figure 3. A Levene's test for homogeneity of variances revealed that young and older adults did not significantly differ in the degree of $\mathrm{HV}$ variability $[F(1,57)=0.64, P=$ $0.43]$. There was a significant interaction effect between age and age group $[F(1,53)=5.80, P=0.02]$, where among older adults, advanced age was associated with smaller HV, yet among young adults, increased age was associated with larger HV. There was no main effect of age, education or an interaction between age group and education (all $P>0.08$ ).

\section{Associations Between HV and Cortisol Levels Measured at Home}

Results of the correlational analyses showed that HV was not associated with any of the three diurnal cortisol measures in young (CAR: $r=-0.02, P=0.91$; average PM cortisol levels: $r$ $=0.09, P=0.69$; maximum-minimum cortisol levels: $r=$ -0.25, $P=0.23$ ), and older adults (CAR: $r=-0.28, P=$ 0.17 ; average $\mathrm{PM}$ cortisol levels: $r=-0.25, P=0.20$; maximum-minimum cortisol levels: $r=0.03, P=0.89$ ).

\section{Associations Between HV and Cortisol Levels Measured at the Testing Environments}

Among older adults, correlational analyses performed between $\mathrm{HV}$ and AUCg cortisol levels in each testing environment

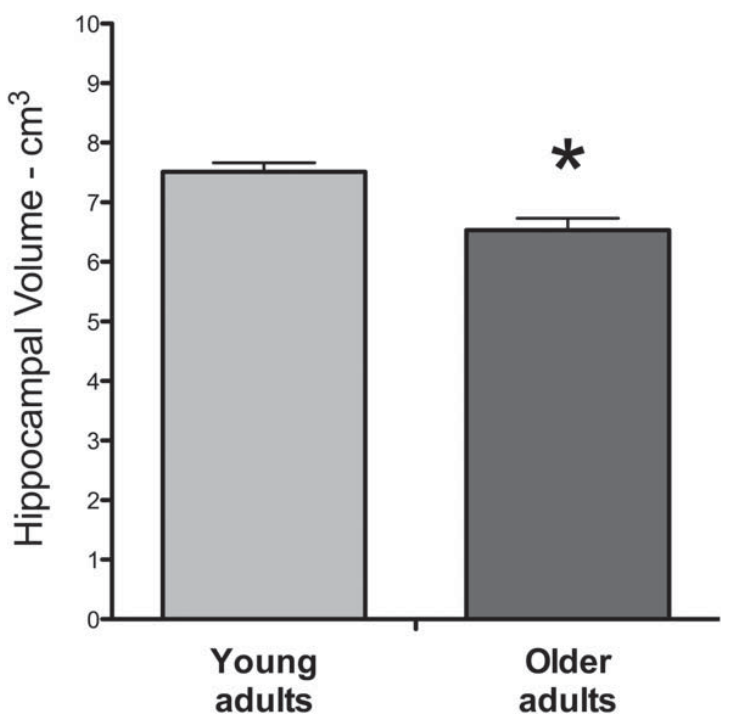

FIGURE 3. older adults.
Total HV (sum of left and right) in young and 
(A) Older Adults

Favouring-Young Condition

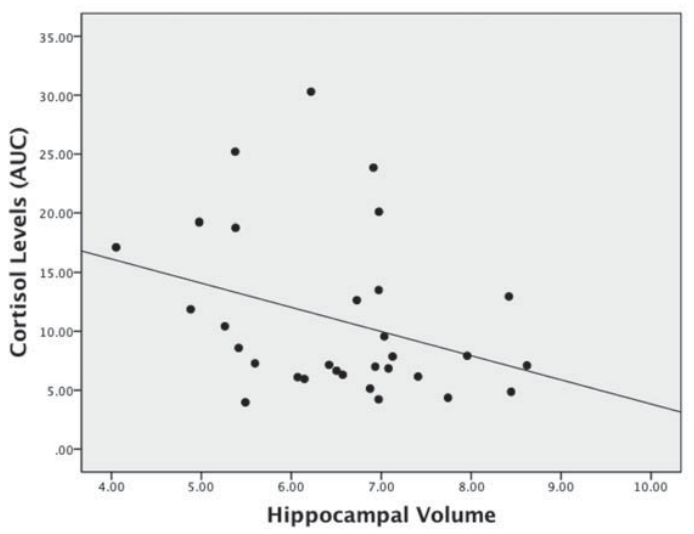

(B) Young Adults

Favouring-Old Condition

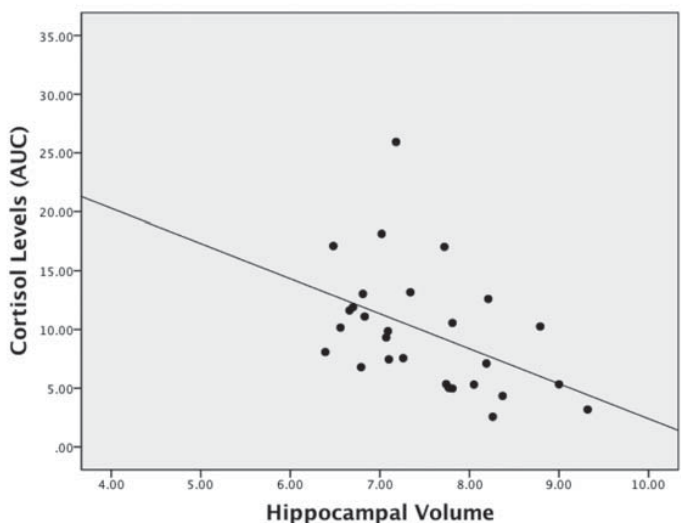

FIGURE 4. Scatterplots demonstrating the significant correlations between HV and cortisol levels when older and young adults are tested in their non-favoring environments.

revealed a significant negative correlation between HV and cortisol AUCg levels in the Favoring-Young environment (see Table 2 and Fig. 4A), so that smaller HV was associated with larger cortisol secretion. In contrast, no significant correlation was observed between $\mathrm{HV}$ and cortisol levels in the Favoring-Old environment (Fig. 5A). Correlational analyses in young adults showed the same pattern as a function of favoring/unfavoring testing conditions; smaller $\mathrm{HV}$ was associated with larger cortisol secretion in the Favoring-Old environment (see Table 2 and Fig. 4B), while there was no significant correlation observed between $\mathrm{HV}$ and cortisol levels in the Favoring-Young environment (Fig. 5B). This demonstrates that in each population, smaller HV was associated with a higher stress response in the non-favoring environment.

To determine whether in each testing environment, the reported correlations differ between the age groups, we compared the correlation coefficients using the Fisher $r-z$ transfor- mation test for comparisons of correlation coefficients. Results revealed that coefficients significantly differed for the FavoringYoung condition $(z=2.94, P=0.003)$, but not for the Favoring-Old condition $(z=0.99, P=0.32)$.

In contrast to analyses performed using cortisol AUCg, analyses using AUCi did not reveal any significant associations between HV and AUCi for young and older adults in both conditions (as can be seen in Table 3).

\section{DISCUSSION}

The results of this study show that when young and older adults are tested in an unfavorable (stressful) environment for their age group, small HV is associated with higher levels of
(A) Older Adults

Favouring-Old Condition

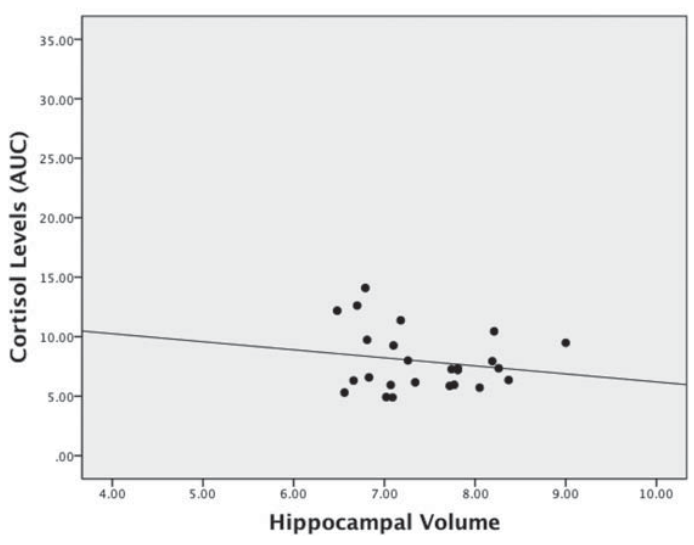

(B) Young Adults

Favouring-Young Condition

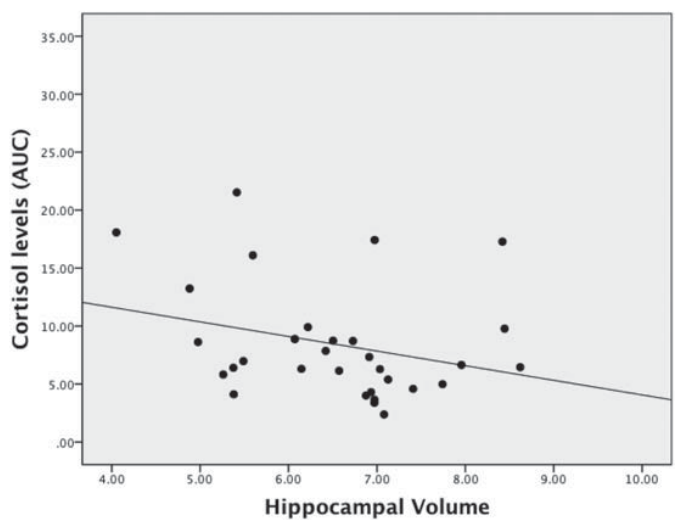

FIGURE 5. Scatterplots demonstrating the non-significant correlations between HV and cortisol levels when older and young adults are tested in their favoring environments. 
TABLE 1.

Descriptive Statistical Values for All Cortisol Measures at the (A) Favoring-Old Condition and (B) Favoring-Young Condition

\begin{tabular}{|c|c|c|c|c|c|c|}
\hline & Sample $1 M$ (SE) & Sample $2 M$ (SE) & Sample $3 M$ (SE) & Sample $4 M$ (SE) & Sample $5 M$ (SE) & Sample $6 M$ (SE) \\
\hline \multicolumn{7}{|c|}{ Favoring-Old condition } \\
\hline Young adults & $0.30(0.03)$ & $0.27(0.03)$ & $0.25(0.03)$ & $0.21(0.02)$ & $0.19(0.02)$ & $0.18(0.02)$ \\
\hline Older adults & $0.25(0.03)$ & $0.21(0.02)$ & $0.21(0.02)$ & $0.21(0.02)$ & $0.20(0.02)$ & $0.19(0.02)$ \\
\hline \multicolumn{7}{|c|}{ Favoring-Young condition } \\
\hline Young adults & $0.16(0.02)$ & $0.15(0.02)$ & $0.15(0.03)$ & $0.13(0.02)$ & $0.11(0.01)$ & \\
\hline Older adults & $0.17(0.02)$ & $0.17(0.02)$ & $0.16(0.02)$ & $0.16(0.02)$ & $0.14(0.02)$ & \\
\hline
\end{tabular}

AUCg cortisol levels ("now you see it"). By contrast, when the same sample of young and older adults is tested in a favorable (less stressful) environment for their age group, HV is not associated with AUCg cortisol levels ("now you do not").

Moreover, HV was not associated with the three measures of basal cortisol levels sampled at home (CAR, average PM cortisol, maximum-minimum cortisol levels) among young and older adults. Consequently, this study provides additional evidence that significant associations between HV and cortisol levels might depend on participants' stress reactivity to the testing environment. The lack of associations between HV and homebased basal measures contradict some studies while they support others (Frodl and O'Keane, 2013). Studies that have found significant associations between $\mathrm{HV}$ and basal cortisol levels vary substantially in methods of cortisol sampling (urinary, blood and/or salivary) and the portion of basal cortisol that was associated with HV (24-h sampling, CAR, awakening, or evening cortisol levels). Among these studies, very few have shown consistent findings while using the same sampling methods and age groups (Beresford et al., 2006; O'Hara et al., 2007; Pruessner et al., 2007; Bruehl et al., 2009; Kremen et al., 2010). It is thus possible that these discrepant data be explained by important variations in testing conditions associated with sampling of "basal" cortisol levels. For example, Beresford et al. (2006) reported a significant association between smaller $\mathrm{HV}$ and higher awakening cortisol levels among middle-aged and older adults. However, in this study, samples for morning cortisol levels were collected on the day of the MRI scan, thus leading to the possibility that anticipation of the MRI session could have led to increased morning cortisol levels in participants, and to the significant association between morning cortisol levels and HV.

Consistent with previous findings, we found that $\mathrm{HV}$ of older adults was smaller than that of young adults; however, a wide range of inter-individual $\mathrm{HV}$ variability was found in both age groups (Lupien et al., 2007a). In both age groups, individuals with smaller $\mathrm{HV}$ were more reactive to the testing environment, implying that correlations between $\mathrm{HV}$ and cortisol levels are not a function of HV threshold effects, where a given $\mathrm{HV}$ leads to increased stress reactivity [see Lupien et al., (2007a) for a review of this model]. Instead, the results suggest a potential stress resistance threshold as a function of $\mathrm{HV}$ range reflective of a given age group.

Although the literature is discrepant, several studies on young and older adults report that individuals with small HV present higher basal and reactive cortisol levels (Lupien et al., 1998; Wolf et al., 2002; Vythilingam et al., 2004; Pruessner et al., 2007). Accordingly, since older adults have smaller HV compared to young individuals, we could have expected older adults to present negative correlations between HV and cortisol levels in both testing environments; instead, the correlation between HV and cortisol levels was only present in their age group-specific unfavorable testing environment. It is however noteworthy that the lack of significant correlations in the favoring conditions (for both age groups) does not imply that associations are absent, but it rather reflects a reduction in the magnitude of observed correlations, depending on the stressfulness of the testing environments. Results on subjective preferences further support our previous findings showing that the unfavorable testing environments were indeed non-optimal for

TABLE 2.

Results of Correlational Analyses Between HV and AUCg Cortisol Levels in the Favoring-Young and the Favoring-Old Conditions in Young and Older Adults

\begin{tabular}{|c|c|c|c|c|}
\hline \multirow[b]{2}{*}{ Favoring-Young condition } & \multicolumn{2}{|c|}{ Young adults } & \multicolumn{2}{|c|}{ Older adults } \\
\hline & $\begin{array}{c}\text { Unadjusted model } \\
r=-0.18, P=0.40\end{array}$ & $\begin{array}{c}\text { Adjusted model } \\
r=-0.19, P=0.383\end{array}$ & $\begin{array}{c}\text { Unadjusted model } \\
r=-0.330, P=0.070\end{array}$ & $\begin{array}{c}\text { Adjusted model } \\
r=-\mathbf{0 . 3 8}, P=\mathbf{0 . 0 4 3 ^ { * }}\end{array}$ \\
\hline Favoring-Old condition & $r=-0.45, P=0.015^{*}$ & $r=-0.45, P=0.022^{*}$ & $r=-0.29, P=0.120$ & $r=-0.21, P=0.265$ \\
\hline
\end{tabular}

Note: Adjusted models used age and education as covariates. 
TABLE 3.

Results of Correlational Analyses Between HV and AUCi Cortisol Levels in the Favoring-Young and the Favoring-Old Conditions in Young and Older Adults

\begin{tabular}{llllll}
\hline & \multicolumn{2}{c}{ Young adults } & & \multicolumn{2}{c}{ Older adults } \\
\cline { 2 - 3 } & Unadjusted model & Adjusted model & & Unadjusted model & Adjusted model \\
\hline Favoring-Young condition & $r=0.32, P=0.108$ & $r=0.34, P=0.103$ & & $r=0.29, P=0.120$ & $r=-0.01, P=0.953$ \\
Favoring-Old condition & $r=0.17, P=0.404$ & $r=0.13, P=0.538$ & & $r=-0.11, P=0.543$ & $r=-0.15, P=0.425$ \\
\hline
\end{tabular}

Note: Adjusted models used age and education as covariates.

each age group, and that young and older adults preferred the favorable environments for their age group (Sindi et al., 2013). Considering that the same sample of young and older adults were tested in both environments, the current results demonstrate the specific role of the testing environment in modulating associations between $\mathrm{HV}$ and cortisol levels, regardless of age group. It is therefore important to take the testing context into account when studying these associations. Our findings are consistent with Tessner et al.'s (2007) study, where they assessed associations between HV and cortisol levels following pharmacological challenge (hydrocortisone to increase cortisol levels). They found that under baseline conditions, no associations between $\mathrm{HV}$ and cortisol levels were observed, while a significant association was observed post-challenge. Collectively, these findings suggest that to detect associations between $\mathrm{HV}$ and cortisol levels, a sufficiently robust cortisol response is needed, such as in response to the unfavorable testing environments.

Comparisons of the correlation coefficients revealed that for the Favoring-Young condition, coefficients significantly differed between young and older adults, but this was not the case for the Favoring-Old condition. This contrast for the two testing environments may be explained by the time of testing; the Favoring-Old condition was performed in the morning, a period of elevated basal cortisol levels that may have prevented further increases in cortisol levels, especially among young adults who tend to wake up later than older adults, as previously shown (Sindi et al., 2013). In contrast to our results with AUCg, no significant associations were detected between $\mathrm{HV}$ and AUCi. Since AUCg incorporates the change from the ground (baseline sample), it may be a more sensitive measure to detecting the anticipatory response prior to the first sample, which is grounded in zero. Contrary to our expectations, we did not observe a significant cortisol response to the MRI. This however does not contradict previous findings on the stressfulness of the MRI scan. For example, significant differences were found when comparing scanner-naïve and experienced participants, while none were found for within-group comparison, as we did (Tessner et al., 2006). In our current study, there was a trend for an age group effect, where older adults showed higher cortisol levels than young adults before and after the MRI, possibly indicating heightened sensitivity to its stress- ful characteristics. As previously stated, some patient groups are vulnerable to MRI-induced stress and elicit a larger reactive stress response, which is an important confounder (Muehlhan et al., 2011). Older adults may also be a vulnerable group that experiences stress due to anticipating incidental results reflecting pathology (Katz et al., 1994). Suggestions have been proposed to decrease MRI-related stress (e.g., using Mock-MRIs, prior exposure to MRI noises, discussing participants' concerns, and providing detailed information about scanning procedure) (Katz et al., 1994; Lueken et al., 2012; Duncan and Northoff, 2013).

The current findings on significant associations between HV and cortisol levels in specific testing environments may be explained by hippocampal contextualisation of information. To evaluate stressful events, they are contextualised through spatial and temporal recall of past memories and experiences (Eichenbaum, 1997; Moscovitch and Nadel, 1998). Individuals with smaller HV may be less capable of contextualising memories and information, and consequently novel events and environments might be perceived as highly stressful due to erroneous perceptions and interpretations of stressors involved (Pruessner and Baldwin, 2009). It may also be speculated that smaller HV contains less GC receptors leading to sub-optimal negative feedback mechanisms, which results in higher stress responsivity (Herman et al., 2005; Jacobson, 2005).

While the current findings are important and relevant, a few limitations should be noted. First, the small sample size did not allow for subgroup analyses of sex differences. Second, specific sub-regions of the hippocampus were not analysed. Third, our study only measured HV and not volumes of other regions involved in regulating the HPA axis (such as the amygdala or prefrontal cortex), so it is unclear whether the findings are specific to the hippocampus. Fourth, the screening process did not include a medical exam, and instead relied on self-reports of conditions. Additionally, our CAR measure included the awakening and awakening +30 minutes samples, yet it has been suggested that additional measures, including the awakening +60 minutes sample, are important to measure the complete CAR response (Clow et al., 2004). Finally, considering that the Favoring-Young session took place in the afternoon, while the Favoring-Old session was carried out in the morning, the results do not allow us to examine the extent to which time of 
day is a determining factor for our findings. The hippocampus has two types of GC receptors [GC receptors (GR) and mineralocorticoid receptors (MR)], which are differentially occupied at different time periods of the day (in the morning, $67-74 \%$ of MRs are occupied, and $74 \%$ of GRs, whereas in the afternoon, $90 \%$ of MRs are occupied, and only $10 \%$ of GRs). It is possible that this difference impacts cortisol reactivity and its association with HV. However, one may speculate that if one pattern of receptor occupation were more likely to reveal significant correlations with $\mathrm{HV}$, it would apply to both age groups, which is not the case in the current study.

In conclusion, this is a compelling study showing that what is considered "basal" cortisol levels in any given study might in fact be a measure of reactive cortisol levels given the nature of the testing environment to which participants are exposed. Consequently, caution should be exercised when assessing associations between $\mathrm{HV}$ and cortisol levels as such findings may be modulated by the nature of the testing environment.

\section{ACKNOWLEDGMENTS}

The authors like to thank Helen Findlay at the Centre for Studies on Human Stress laboratory for conducting the cortisol assays. They would also like to thank the reviewers for their constructive feedback and comments to previous versions of the manuscript.

\section{REFERENCES}

Beresford TP, Arciniegas DB, Alfers J, Clapp L, Martin B, Beresford HF, Du Y, Liu D, Shen D, Davatzikos C, Laudenslager ML. 2006. Hypercortisolism in alcohol dependence and its relation to hippocampal volume loss. J Stud Alcohol 67:861-867.

Bremner JD. 2006. Traumatic stress: Effects on the brain. Dialogues Clin Neurosci 8:445-461.

Bruehl H, Wolf OT, Convit A. 2009. A blunted cortisol awakening response and hippocampal atrophy in type 2 diabetes mellitus. Psychoneuroendocrinology 34:815-821.

Clow A, Hucklebridge F, Thorn L. 2010. The cortisol awakening response in context. Int Rev Neurobiol 93:153-175.

Clow A, Thorn L, Evans P, Hucklebridge F. 2004. The awakening cortisol response: Methodological issues and significance. Stress 7: $29-37$.

Collins DL, Neelin P, Peters TM, Evans AC. 1994. Automatic 3d intersubject registration of $\mathrm{Mr}$ volumetric data in standardized talairach space. J Computer Assist Tomogr 18:192-205.

Collip D, Habets P, Marcelis M, Gronenschild E, Lataster T, Lardinois M, Nicolson NA, Myin-Germeys I. 2013. Hippocampal volume as marker of daily life stress sensitivity in psychosis. Psychol Med 43:1377-1387.

de Kloet ER, Joels M, Holsboer F. 2005. Stress and the brain: From adaptation to disease. Nat Rev Neurosci 6:463-475.

Dickerson SS, Kemeny ME. 2004. Acute stressors and cortisol responses: A theoretical integration and synthesis of laboratory research. Psychol Bull 130:355-391.
Duncan NW, Northoff G. 2013. Overview of potential procedural and participant-related confounds for neuroimaging of the resting state. J Psychiatry Neurosci 38:84-96.

Eichenbaum H. 1997. How does the brain organize memories? Science 277:330-332.

Evans AC, Collins DL, Millst SR, Brown ED, Kelly RL, Peters TM. 1994. 3D statistical neuroanatomical models from 305 MRI volumes. IEEE Nucl Sci Symp Med Imaging Conference 3:1813-1817.

Fries E, Dettenborn L, Kirschbaum C. 2009. The cortisol awakening response (CAR): Facts and future directions. Int J Psychophysiol 72:67-73.

Frodl T, O'Keane V. 2013. How does the brain deal with cumulative stress? A review with focus on developmental stress, HPA axis function and hippocampal structure in humans. Neurobiol Dis 52: $24-37$.

Gold SM, Kern KC, O'Connor MF, Montag MJ, Kim A, Yoo YS, Giesser BS, Sicotte NL. 2010. Smaller cornu ammonis 2-3/dentate gyrus volumes and elevated cortisol in multiple sclerosis patients with depressive symptoms. Biol Psychiatry 68:553-559.

Gunduz-Bruce H, Szeszko PR, Gueorguieva R, Ashtari M, Robinson DG, Kane JM, Bilder RM. 2007. Cortisol levels in relation to hippocampal sub-regions in subjects with first episode schizophrenia. Schizophr Res 94:281-287.

Herman JP, Ostrander MM, Mueller NK, Figueiredo H. 2005. Limbic system mechanisms of stress regulation: Hypothalamopituitary-adrenocortical axis. Prog in Neuropsychopharmacol Biol Psychiatry 29:1201-1213.

Jacobson L. 2005. Hypothalamic-pituitary-adrenocortical axis regulation. Endocrinol Metab Clin North Am 34:271-292, vii.

Karl A, Schaefer M, Malta LS, Dorfel D, Rohleder N, Werner A. 2006. A meta-analysis of structural brain abnormalities in PTSD. Neurosci Biobehav Rev 30:1004-1031.

Katz RC, Wilson L, Frazer N. 1994. Anxiety and its determinants in patients undergoing magnetic resonance imaging. J Behav Ther Exp Psychiatry 25:131-134.

Kaymak SU, Demir B, Senturk S, Tatar I, Aldur MM, Ulug B. 2010. Hippocampus, glucocorticoids and neurocognitive functions in patients with first-episode major depressive disorders. Eur Arch Psychiatry Clin Neurosci 260:217-223.

Kirschbaum C, Pirke KM, Hellhammer DH. 1993. The 'Trier Social Stress Test'-a tool for investigating psychobiological stress responses in a laboratory setting. Neuropsychobiology 28 $76-81$.

Knoops AJ, Gerritsen L, van der Graaf Y, Mali WP, Geerlings MI. 2010. Basal hypothalamic pituitary adrenal axis activity and hippocampal volumes: The SMART-Medea study. Biol Psychiatry 67: $1191-1198$

Kremen WS, O’Brien RC, Panizzon MS, Prom-Wormley E, Eaves LJ, Eisen SA, Eyler LT, Hauger RL, Fennema-Notestine C, Fischl B, Grant MD, Hellhammer DH, Jak AJ, Jacobson KC, Jernigan TL, Lupien SJ, Lyons MJ, Mendoza SP, Neale MC, Seidman LJ, Thermenos HW, Tsuang MT, Dale AM, Franz CE. 2010. Salivary cortisol and prefrontal cortical thickness in middle-aged men: A twin study. Neuroimage 53:1093-1102.

Lueken U, Muehlhan M, Evens R, Wittchen HU, Kirschbaum C. 2012. Within and between session changes in subjective and neuroendocrine stress parameters during magnetic resonance imaging: A controlled scanner training study. Psychoneuroendocrinology 37: 1299-1308.

Lupien SJ, de Leon M, de Santi S, Convit A, Tarshish C, Nair NP, Thakur M, McEwen BS, Hauger RL, Meaney MJ. 1998. Cortisol levels during human aging predict hippocampal atrophy and memory deficits. Nat Neurosci 1:69-73.

Lupien SJ, Evans A, Lord C, Miles J, Pruessner M, Pike B, Pruessner JC. 2007a. Hippocampal volume is as variable in young as in older adults: Implications for the notion of hippocampal atrophy in humans. Neuroimage 34:479-485. 
Lupien SJ, Gaudreau S, Tchiteya BM, Maheu F, Sharma S, Nair NP, Hauger RL, McEwen BS, Meaney MJ. 1997. Stress-induced declarative memory impairment in healthy elderly subjects: Relationship to cortisol reactivity. J Clin Endocrinol Metab 82:20702075.

Lupien SJ, Maheu F, Tu M, Fiocco A, Schramek TE. 2007b. The effects of stress and stress hormones on human cognition: Implications for the field of brain and cognition. Brain Cogn 65:209-237.

Lupien SJ, McEwen BS. 1997. The acute effects of corticosteroids on cognition: Integration of animal and human model studies. Brain Res Brain Res Rev 24:1-27.

MacLullich AMJ, Deary IJ, Starr JM, Ferguson KJ, Wardlaw JM, Seckl JR. 2005. Plasma cortisol levels, brain volumes and cognition in healthy elderly men. Psychoneuroendocrinology 30:505-515.

Mason JW. 1968. A review of psychoendocrine research on the pituitaryadrenal cortical system. Psychosom Med 30:Suppl:576-607.

McEwen BS. 2007. Physiology and neurobiology of stress and adaptation: Central role of the brain. Physiol Rev 87:873-904.

Mondelli V, Pariante CM, Navari S, Aas M, D'Albenzio A, Di Forti M, Handley R, Hepgul N, Marques TR, Taylor H, Papadopoulos AS, Aitchison KJ, Murray RM, Dazzan P. 2010. Higher cortisol levels are associated with smaller left hippocampal volume in firstepisode psychosis. Schizophr Res 119:75-78.

Moscovitch M, Nadel L. 1998. Consolidation and the hippocampal complex revisited: In defense of the multiple-trace model - Discussion point. Curr Opin Neurobiol 8:297-300.

Muehlhan M, Lueken U, Wittchen HU, Kirschbaum C. 2011. The scanner as a stressor: Evidence from subjective and neuroendocrine stress parameters in the time course of a functional magnetic resonance imaging session. Int J Psychophysiol 79:118-126.

Nicolson N, Storms C, Ponds R, Sulon J. 1997. Salivary cortisol levels and stress reactivity in human aging. J Gerontol A Biol Sci Med Sci 52:M68-M75.

O’Brien JT, Lloyd A, McKeith I, Gholkar A, Ferrier N. 2004. A longitudinal study of hippocampal volume, cortisol levels, and cognition in older depressed subjects. Am J Psychiatry 161:2081-2090.

O'Hara R, Schroder CM, Mahadevan R, Schatzberg AF, Lindley S, Fox S, Weiner M, Kraemer HC, Noda A, Lin X, Gray HL, Hallmayer JF. 2007. Serotonin transporter polymorphism, memory and hippocampal volume in the elderly: Association and interaction with cortisol. Mol Psychiatry 12:544-555.

Pruessner JC, Kirschbaum C, Meinlschmid G, Hellhammer DH. 2003. Two formulas for computation of the area under the curve represent measures of total hormone concentration versus timedependent change. Psychoneuroendocrinology 28:916-931.

Pruessner JC, Li LM, Serles W, Pruessner M, Collins DL, Kabani N, Lupien S, Evans AC. 2000. Volumetry of hippocampus and amygdala with high-resolution MRI and three-dimensional analysis software: Minimizing the discrepancies between laboratories. Cereb Cortex 10:433-442.

Pruessner JCW, S; Baldwin, M. W. 2009. Stress of self esteem.In: Fink G, editor. Stress Consequences: Mental, Neuropsychological and Socioeconomic. Oxford, UK: Academic Press. pp 53-57.

Pruessner M, Pruessner JC, Hellhammer DH, Pike GB, Lupien SJ. 2007. The associations among hippocampal volume, cortisol reactivity, and memory performance in healthy young men. Psychiatry Res 155:1-10.

Sindi S, Fiocco AJ, Juster RP, Pruessner J, Lupien SJ. 2013. When we test, do we stress? Impact of the testing environment on cortisol secretion and memory performance in older adults. Psychoneuroendocrinology 38:1388-1396.

Sled JG, Zijdenbos AP, Evans AC. 1998. A nonparametric method for automatic correction of intensity nonuniformity in MRI data. IEEE Trans Med Imaging 17:87-97.

Swaab DF, Bao AM, Lucassen PJ. 2005. The stress system in the human brain in depression and neurodegeneration. Ageing Res Rev 4:141-194.

Tessner KD, Walker EF, Dhruv SH, Hochman K, Harnann S. 2007. The relation of cortisol levels with hippocampus volumes under baseline and challenge conditions. Brain Res 1179:70-78.

Tessner KD, Walker EF, Hochman K, Hamann S. 2006. Cortisol responses of healthy volunteers undergoing magnetic resonance imaging. Hum Brain Mapp 27:889-895.

Van Petten C. 2004. Relationship between hippocampal volume and memory ability in healthy individuals across the lifespan: Review and meta-analysis. Neuropsychologia 42:1394-1413.

Vythilingam M, Vermetten E, Anderson GM, Luckenbaugh D, Anderson ER, Snow J, Staib LH, Charney DS, Bremner JD. 2004. Hippocampal volume, memory, and cortisol status in major depressive disorder: Effects of treatment. Biol Psychiatry 56:101112.

Wolf OT, Convit A, de Leon MJ, Caraos C, Qadri SF. 2002. Basal hypothalamo-pituitary-adrenal axis activity and corticotropin feedback in young and older men: Relationships to magnetic resonance imaging-derived hippocampus and cingulate gyrus volumes. Neuroendocrinology 75:241-249. 